$16^{\text {th }}$ International Congress of Metrology, 08004 (2013)

DOI: $10.1051 /$ metrology/201308004

(C) Owned by the authors, published by EDP Sciences, 2013

\title{
La mesure de la microrugosité en environnement industriel : Cas des pièces mécaniques
}

\author{
Bertrand Leroy ${ }^{1}$ \\ ${ }^{1}$ PSA Peugeot-Citroën, Métrologie Centrale Mécanique, 18 rue des Fauvelles 92250 La Garenne-Colombes, \\ France
}

Résumé. L'amélioration de la performance des moteurs à combustion interne passe par la réduction significative des pertes par frottements mécaniques. Cette réduction est en général obtenue par la réduction et une meilleure maitrise des états de surface. II est désormais nécessaire de maitriser des spécifications d'états de surface inférieures à $0,25 \mu \mathrm{m}$ et ce dans un environnement industriel moins favorables qu'en laboratoire. Cette étude se base sur deux pièces : le vilebrequin et les chemises de carter cylindres.

\begin{abstract}
Improving the performance of internal combustion engines pass by significantly reducing mechanical losses by friction. This reduction is often obtained by reduction and better control of surface textures. The need to master specifications of surface textures under 0.25 microns is now increasingly common and this, in an industrial environment, with conditions less favourable than in the laboratory. This study uses two examples: crankshaft and cylinder block liners.
\end{abstract}

\section{Mesure de rugosité sur les paliers de vilebrequin}

\subsection{Caractéristiques mesurées}

La mesure de la rugosité sur les paliers d'un vilebrequin se fait le long de génératrices de ces surfaces cylindriques.

Les caractéristiques à suivre sont, pour la rugosité, un paramètre d'amplitude moyenne de type $\mathrm{R}$ ou $\mathrm{Rz}$ et un taux de longueur portante spécifié en Rk et Rpk.

Les valeurs sont dans le cas étudié de 0,8 pour $\mathrm{Rz}$, 0,25 pour Rk et 0,1 pour Rpk.

Les surfaces mesurées sont obtenues par toilage.

\subsection{Problématique :}

Valider le moyen de mesure dans son contexte atelier et déterminer la méthode de mesure permettant d'obtenir une incertitude de mesure conforme aux règles internes à PSA.

\subsection{Contraintes :}

Le fournisseur des machines à mesurer était nouveau pour PSA. Les deux machines de mesures devaient être situées dans une métrologie d'atelier qui ne dispose pas de dalle isolée. L'accessibilité aux surfaces à mesurer est difficile en raison de la géométrie complexe de la pièce sachant que la gamme de contrôle devait être fortement automatisée.

\subsection{Les essais et mesures}

Suite à l'installation des machines dans l'atelier, l'usine a constaté immédiatement une forte différence sur les résultats de mesure entre ces nouvelles machines et les résultats de sa propre métrologie centrale installée dans un environnement particulièrement favorable et avec des moyens de mesure connus.

Elle a donc sollicité notre métrologie de référence pour l'aider dans ses investigations.

Il a été décidé en relation avec le fournisseur des machines d'effectuer une série d'essais à l'aide de deux étalons spécifiques et d'un vilebrequin servant de référence et ce dans l'ensemble des configurations de mesure possibles. De plus il a été décidé d'intercomparer ces nouvelles machines avec l'ensemble des matériels concurrents dont nous 
disposons dans l'usine et dans notre métrologie de référence.

Jusqu'à neuf appareils ont été intercomparés, six à l'usine et trois en métrologie de référence. Sur les machines à valider, nous avons testé plusieurs configurations de mesure : Mesure suivant l'axe $\mathrm{X}$ et suivant l'axe $\mathrm{Y}$, angle du stylet à $0^{\circ}$ ou à $90^{\circ}$ par rapport à l'axe de déplacement, capteur standard et capteur spécial (donnant une meilleure accessibilité à la surface à mesurer).

Le tableau 1 montre les configurations testées par localisation et famille d'appareil.

La famille de machine $\mathrm{N}^{\circ} 3$ est celle que nous devions valider et comporte deux machines identiques.

La configuration retenue par le projet au début de l'étude était: mesure suivant $\mathrm{Y}$ à $90^{\circ}$, capteur spécial, stylet avec pointe courte, 3 mesures par génératrice, trois génératrices par cylindre à mesurer.

\begin{tabular}{|c|c|c|c|c|c|c|c|c|}
\hline & $\begin{array}{l}\text { 言 } \\
\text { 乭 } \\
\text { 至 }\end{array}$ & $\begin{array}{l}\text { 䔍 } \\
\text { 空 }\end{array}$ & 穿 & \multicolumn{3}{|c|}{ 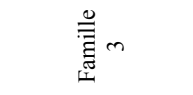 } & 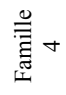 & 营 \\
\hline $\begin{array}{l}\text { Métro. de } \\
\text { référence }\end{array}$ & 1 & & & & & & 2 & \\
\hline $\begin{array}{c}\text { Métro. } \\
\text { centrale } \\
\text { usine }\end{array}$ & & 1 & & & & & & \\
\hline $\begin{array}{c}\text { Métro. } \\
\text { d'atelier }\end{array}$ & & & 1 & \multicolumn{3}{|c|}{2} & & 2 \\
\hline $\begin{array}{c}\text { Environne } \\
\text { ment }\end{array}$ & ++ & + & - & \multicolumn{3}{|c|}{ - } & ++ & - \\
\hline $\begin{array}{l}\text { Axe de } \\
\text { mesure }\end{array}$ & $\begin{array}{l}\dot{0} \\
\ddot{x} \\
x\end{array}$ & 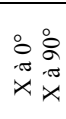 & 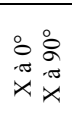 & $\begin{array}{l}0 \\
\vdots \\
-\pi \\
\lambda\end{array}$ & $\begin{array}{l}\vdots \\
\vdots \\
\check{x} \\
\check{x}\end{array}$ & $\begin{array}{l}0 \\
0 \\
\pi \\
x\end{array}$ & $\begin{array}{ll}0 & 0 \\
0 & 0 \\
\pi & -\pi \\
x & \nearrow\end{array}$ & $\begin{array}{l}: \\
0 \\
0 \\
-\pi \\
x \\
x \\
x \\
x\end{array}$ \\
\hline Capteurs & 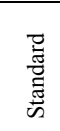 & 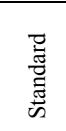 & 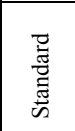 & 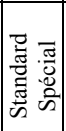 & 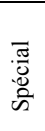 & 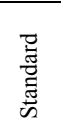 & 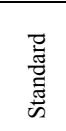 & 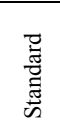 \\
\hline $\begin{array}{c}\text { Décalage } \\
\text { en } Z \text { de la } \\
\text { pointe du } \\
\text { stylet }\end{array}$ & 䓂 & 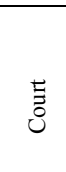 & 音 & 音 & 言 & 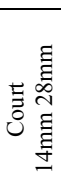 & 音 & 䓂 \\
\hline
\end{tabular}

Tableau 1: Configurations de mesure testées

L'étude permettant de valider la machine et la méthode de mesure s'est faite en quatre étapes :

- Evaluation des bruits de fond

- Mesure de deux étalons de type D

- Intercomparaison de notre vilebrequin de référence.

- Evaluation des incertitudes

\subsubsection{Bruit de fond statique :}

La mesure du bruit de fond statique permet d'évaluer les conditions environnementales et la réponse de la chaine de mesure à celles-ci. En complément, une étude complète à l'aide d'accéléromètres a été menée sur les machines à valider, par notre laboratoire spécialisé dans ce domaine.

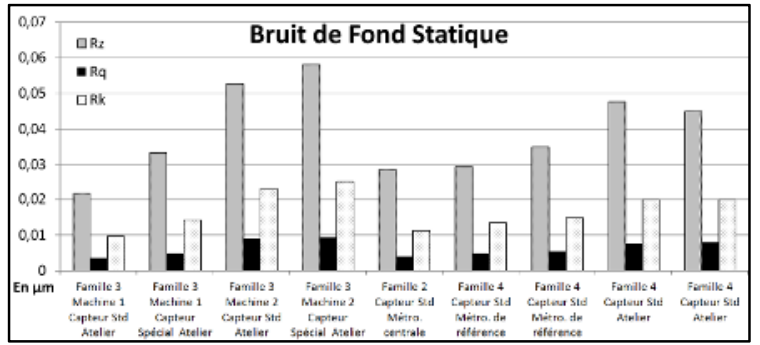

Figure 1 : Mesures de bruit de fond statique

Premier constat :

Malgré un environnement défavorable, le bruit de fond des nouvelles machines est du même ordre de grandeur que pour la métrologie centrale d'usine (Famille 2). Notre appareil de référence (Famille 1) n'est pas inclus car il ne peut faire que du bruit de fond dynamique.

\section{Deuxième constat :}

Une de nos deux machines de la famille $\mathrm{N}^{\circ} 3$ était moins bien placée que l'autre. Cela nous a permis de détecter un problème d'amortisseur actif sur cette machine qui a été réglé.

\section{Troisième constat :}

Le capteur spécial que nous souhaitions utiliser est plus sensible aux vibrations.

\subsubsection{Bruit de fond dynamique :}

En plus du bruit de fond statique le bruit de fond dynamique permet de connaitre l'impact du déplacement de la tête de mesure.

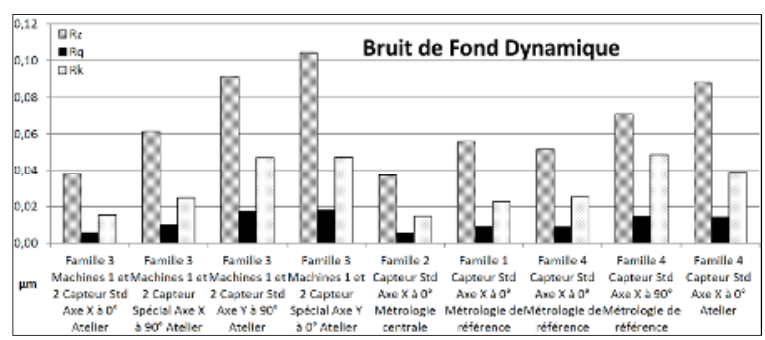

$\underline{\text { Figure 2 }}$ : Mesures de bruit de fond dynamique

\section{Premier constat :}

La mesure suivant l'axe $\mathrm{Y}$ à $90^{\circ}$ est très défavorable. Le niveau de bruit de fond dynamique a été jugé incompatible avec le niveau d'exigence pour cette pièce. Il s'agissait de la configuration qui avait été retenue avant le début de cette étude. 


\section{Deuxième constat :}

Les valeurs de bruit de fond en dynamique se dégradent en général d'au moins 30\% par rapport au bruit de fond statique allant parfois du simple au double.

\subsubsection{Troisième constat :}

Notre meilleur appareil en métrologie de référence n'est pas bien placé : après évaluation nous constatons que sa résolution qui est supérieure à celle des autres appareils, du fait de la technologie de son capteur, ne permet pas d'atteindre des valeurs équivalentes à celles d'appareils disposant d'une résolution plus faible. On constatera ensuite que ce défaut n'a pas beaucoup d'impact sur la mesure des étalons et du vilebrequin de référence.

\subsubsection{Conclusions de l'étude sur le bruit de fond :}

Les machines à valider ont, dans certaines configurations et malgré un environnement défavorable, un bruit de fond compatible avec les mesures à effectuer.

La configuration retenue au départ a été rejetée dès le début de l'étude. Malgré tout nous effectuerons quand même des mesures dans cette configuration sur les étalons et le vilebrequin servant de référence, avec la même conclusion.

\subsubsection{Mesure de l'étalon $N^{\circ} 1$ :}

Il s'agit d'un étalon de type D1 (Suivant ISO 5436-1) de valeurs de $\mathrm{Rz} R k$ et Rpk proches du double de l'exigence du vilebrequin. Cet étalon a été certifié par le laboratoire du PTB (Physikalisch-Technische Bundesanstalt).

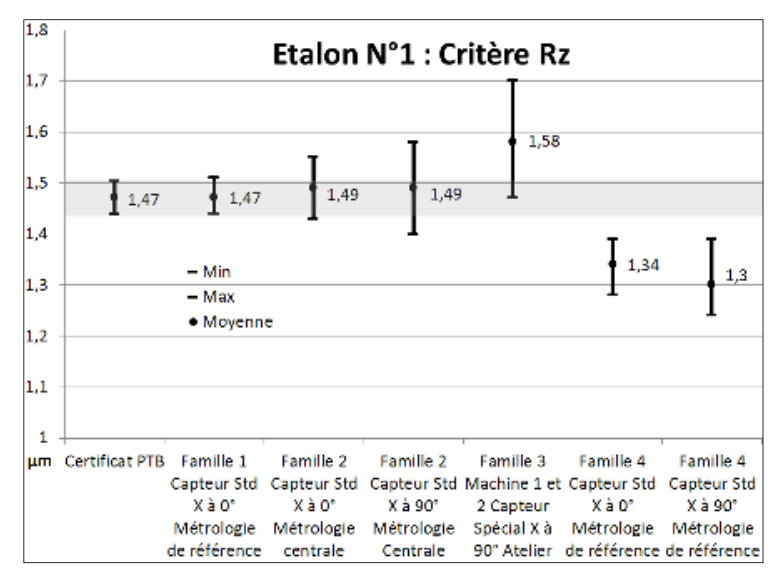

$\underline{\text { Figure } 3}$ : Mesure du critère Rz sur l'étalon $\mathrm{N}^{\circ} 1$

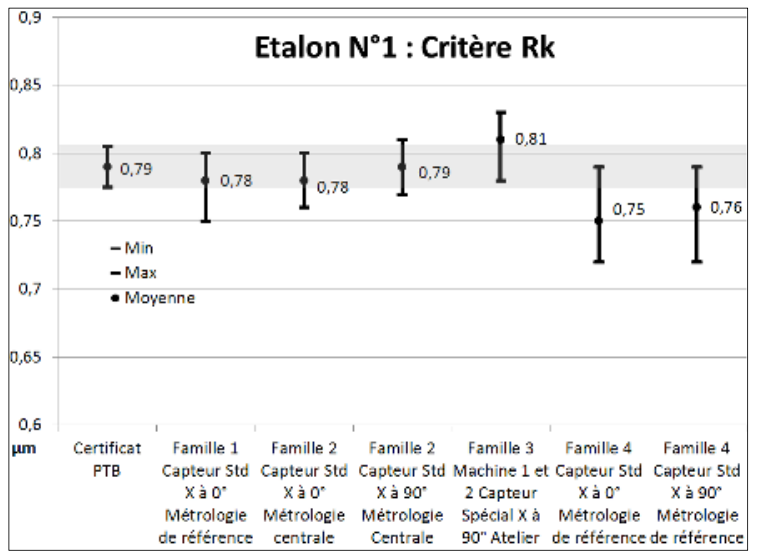

$\underline{\text { Figure } 4}$ : Mesure du critère Rk sur l'étalon $\mathrm{N}^{\circ} 1$

\section{Premier constat :}

Notre machine de référence affiche des résultats similaires au certificat d'étalonnage pour le critère $\mathrm{Rz}$ et très proches pour le $\mathrm{Rk}$.

La machine de référence de l'usine est aussi très proche du résultat attendu. Quand elle mesure à $90^{\circ}$ la dispersion augmente sans impact sur la moyenne pour le Rz.

\section{Deuxième constat :}

La famille $\mathrm{N}^{\circ} 4$ de nos appareils de mesure donne des résultats sous évalués. Ce défaut nous était déjà connu et n'a été que confirmé par cette étude. Il est engendré par une spécificité électronique de ces machines. Pour les spécifications supérieures au micron son impact est négligeable.

\section{Troisième constat :}

Pour la Famille $\mathrm{N}^{\circ} 3$, après la disqualification de l'axe $\mathrm{Y}$ comme axe de mesure, la configuration la plus favorable pour l'accessibilité à la pièce est l'axe $\mathrm{X}$ à $90^{\circ}$ avec le capteur spécial. On constate que cette configuration donne des résultats fortement décalés par rapport aux valeurs de l'étalon.

\section{Quatrième constat :}

L'évaluation des différentes machines montre des résultats plus proches au niveau du critère Rk ce qui était prévisible, malgré cela les tendances sont les mêmes que pour le $\mathrm{Rz}$ qui est plus sensible aux perturbations.

\subsubsection{Mesure de l'étalon $N^{\circ} 2$ :}

Il s'agit d'un étalon de type D1 avec des valeurs plus proches des valeurs du vilebrequin que celles de l'étalon précédent. Il est réalisé par un fournisseur différent. Il est également certifié par le PTB. 
Pour cet étalon nous ne présenterons pas en détail les résultats de mesure.

\section{Premier constat :}

Les résultats sur cet étalon sont majoritairement moins bons que pour le précédent en particulier pour le paramètre Rk. Après étude minutieuse de cet étalon, nous constatons que la pente moyenne de son profil est bien supérieure à celle du précédent et représente donc une difficulté particulière pour les machines de mesure en particulier à $90^{\circ}$.

\section{Deuxième constat :}

Notre machine de référence n'est pas meilleure sur cet étalon que d'autres machines

\section{Troisième constat :}

La famille $\mathrm{N}^{\circ} 4$ est toujours décalée.

\section{Quatrième constat :}

Pour la famille $\mathrm{N}^{\circ} 3$ parmi les cinq configurations testées celles que nous retiendrons finalement (stylets $14 \mathrm{~mm}$ et $28 \mathrm{~mm}$ ) sont assez bien placées même si nous verrons que pour la configuration avec le stylet avec la pointe décalée de $28 \mathrm{~mm}$ la bonne qualité de la valeur trouvée est due probablement à la déformation du stylet et pas à la qualité de la mesure. La mesure à $90^{\circ}$ avec le capteur spécial est à nouveau mal placée.

\subsubsection{Conclusions sur la mesure des étalons type $D$ :}

La mesure de ces deux étalons nous a permis de montrer que la configuration de mesure à $90^{\circ}$ avec le capteur spécial ne pouvait être retenue. Nous verrons que ce constat sera identique sur le vilebrequin.

Nous avons aussi pu constater qu'il n'est pas simple de choisir un étalon et que deux étalons qui semblent assez proches peuvent amener à des conclusions assez différentes.

\subsubsection{Mesure du vilebrequin de référence.}

Nous avons passé beaucoup de temps à mesurer ce vilebrequin, plusieurs centaines de mesures ont été réalisés et analysées.

Nous avons pris le parti de considérer le meilleur appareil de notre métrologie de référence comme donnant la valeur vraie du vilebrequin et ce pour deux raisons: il s'agit de l'appareil que nous considérons comme le plus stable et qui dispose du meilleur environnement. C'est de plus celui avec lequel nous avons effectué le plus de mesures.

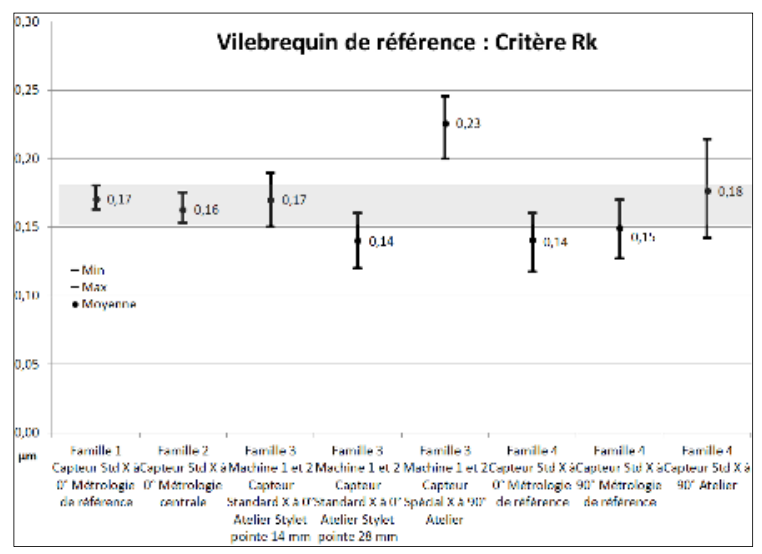

Figure 5 : Mesure du critère Rk sur le vilebrequin

\section{Premier constat :}

L'appareil de la métrologie centrale de l'usine, dans sa meilleure configuration est proche de celui de la métrologie de référence bien qu'un peu inférieur mais dans l'incertitude de répétabilité de positionnement.

\section{Deuxième constat :}

La famille $\mathrm{N}^{\circ} 4$ est encore inférieure aux autres, sachant que l'appareil avec sa configuration la plus défavorable et dans l'environnement le moins maitrisé donne en moyenne la «bonne » valeur au prix cependant d'une dispersion des résultats particulièrement importante.

\section{Troisième constat :}

La famille de machine $\mathrm{N}^{\circ} 3$ présente d'excellents résultats dans sa meilleure configuration étudiée (stylet $14 \mathrm{~mm}$ ) et des résultats très en retrait avec la configuration donnant le meilleur accès à la pièce (capteur spécial mesure $\mathrm{X}$ à $90^{\circ}$ ).

Pour la configuration avec le stylet à pointe de 28 $\mathrm{mm}$ nous constatons une atténuation ainsi qu'une légère déformation $\mathrm{du}$ signal. Cela est particulièrement visible sur un étalon sinusoïdal de type C on l'on constate que la partie montante de la sinusoïde est moins inclinée que la partie descendante tout en ayant un résultat conforme au certificat d'étalonnage. Ce défaut est du soit à une déformation $\mathrm{du}$ stylet ou à une mauvaise compensation du décalage de $28 \mathrm{~mm}$ par la machine. 


\subsection{Conclusions des études réalisées sur le vilebrequin :}

Ces résultats nous ont amenés à changer la méthode de mesure pour passer d'une mesure à $90^{\circ}$ avec le capteur spécial à une mesure à $0^{\circ}$ avec le capteur standard.

Cependant cette dernière configuration est très défavorable pour l'accessibilité aux zones à mesurer. Cela nous a obligé à passer d'une gamme de mesure selon trois génératrices à seulement une seule. De plus sur les sept cylindres à mesurer sur notre vilebrequin, quatre sont accessibles par le stylet de $14 \mathrm{~mm}$ et trois par le stylet de $28 \mathrm{~mm}$, cette configuration n'étant pas exempte de problèmes.

Suite à ce changement il a été nécessaire d'apporter la preuve qu'en mesurant une génératrice au lieu de trois l'incertitude sur la dispersion des résultats liée à la non homogénéité de la surface ne devenait pas rédhibitoire pour cette méthode de mesure.

\subsection{Calcul des incertitudes de mesure :}

Le calcul des incertitudes de mesure pour les paramètres d'états de surface est une tache relativement ardue du fait du grand nombre de facteurs influençant le résultat de mesure et le fait que certains critères font appels à des algorithmes complexes où la variabilité d'un point sur le résultat final n'est pas toujours calculable analytiquement.

Pour le calcul des incertitudes en états de surface, nous utilisons habituellement la méthode du GUM.

A travers son statut de métrologie de référence pour le domaine mécanique, notre métrologie apporte un éclairage aux autres métrologies de l'entreprise sur les méthodologies, la normalisation et les techniques de mesure.

Aussi dans le cadre de cette étude nous avons recherché une méthode statistique pour prononcer la conformité de la pièce en relation avec la méthode de mesure et les moyens mis en œuvre.

\subsubsection{Méthodes utilisées chez PSA}

La méthode que nous utilisons couramment se base sur le GUM. Nous savons cependant qu'une partie des hypothèses liées à cette norme n'est pas respectée par le calcul que nous faisons qui n'est donc pas complètement exact. Il a cependant le mérite d'exister, chacun sachant qu'il y a toujours une incertitude sur le résultat de ce type de calcul.
Beaucoup de facteurs d'incertitude ne sont pas indépendants les uns des autres. De plus, certains sont très difficiles à déterminer et à modéliser : flexion et louvoiement du stylet, jeux internes au capteur, traitement du signal interne aux machines, logiciels... Sur ce dernier point on constate une augmentation de la différence entre les résultats des différents logiciels, pour un même paramètre et avec les mêmes données d'entrée, qui est proportionnelle à la complexité du calcul et à celle de la topographie mesurée.

Jusqu'à ce jour nous n'avons pas testé les méthodes de Monte-Carlo (MCS) pour les états de surface, la fonction de transfert nous semblant trop complexe à appréhender. Cependant ces méthodes semblent prometteuses dans certains cas.

\subsubsection{Calcul statistique pour juger la conformité d'une pièce.}

Dans le cas de notre vilebrequin nous avons donc cherché une méthode différente basée sur la statistique pour juger la conformité.

L'étude que nous avons menée sur les étalons de type $\mathrm{D}$ et le vilebrequin nous a confirmé dans cette voie car nous avons constaté de nombreuses fois que l'incertitude que nous calculions avec notre méthode habituelle n'était pas cohérente avec les résultats trouvés.

Cette incohérence est probablement due au fait que les sources d'incertitudes ont été intégrées de façon erronée ou partiellement.

Quoi qu'il en soit, identifier toutes les sources d'incertitude nous a semblé trop long et complexe sans garantie de résultat.

\section{Premier constat :}

Dans la majorité des cas, la dispersion des résultats de mesure due à la non homogénéité de la pièce est le facteur principal de variabilité dans le résultat final que nous donnons lors du jugement de la conformité. Dans le cas du vilebrequin, ce constat est un peu moins vrai qu'à l'accoutumée du fait des faibles tolérances.

\section{Deuxième constat :}

La possession d'un étalon ayant une géométrie proche de la pièce à mesurer nous a semblé suffisante pour juger de l'impact du moyen sur la distribution des résultats de mesure, en s'assurant que la méthode de mesure dudit étalon soit proche de celle sur la pièce. 
Nous avons cherché à évaluer cette dispersion et à la comparer au certificat de l'étalon qui nous sert de référence.

Ce constat sera vrai si l'étalon est représentatif de la pièce que l'on étudie.

Evidemment, trouver un étalon certifié qui soit proche de la géométrie à mesurer n'est pas forcement simple. Doivent être pris en considération tout autant les valeurs verticales qu'horizontales de l'étalon, ainsi que l'angle des pentes, la taille et le nombre de motifs...

1.6.3 Analyse des résultats de mesure de l'étalon $N^{\circ} 1$ sur la machine de référence de la métrologie.

Nous avons effectué sur les deux étalons des séries de mesures pour évaluer la dispersion de nos résultats et la comparer à celle du certificat d'étalonnage délivré par le PTB pour ces étalons.

Nous montrons ici les résultats des mesures sur l'étalon $\mathrm{N}^{\circ} 1$.

Nous avons effectué dix mesures complètes de l'étalon conformément au certificat ce qui nous a donné 120 échantillons par critère. Nous avons jugé cet échantillon statistiquement représentatif.

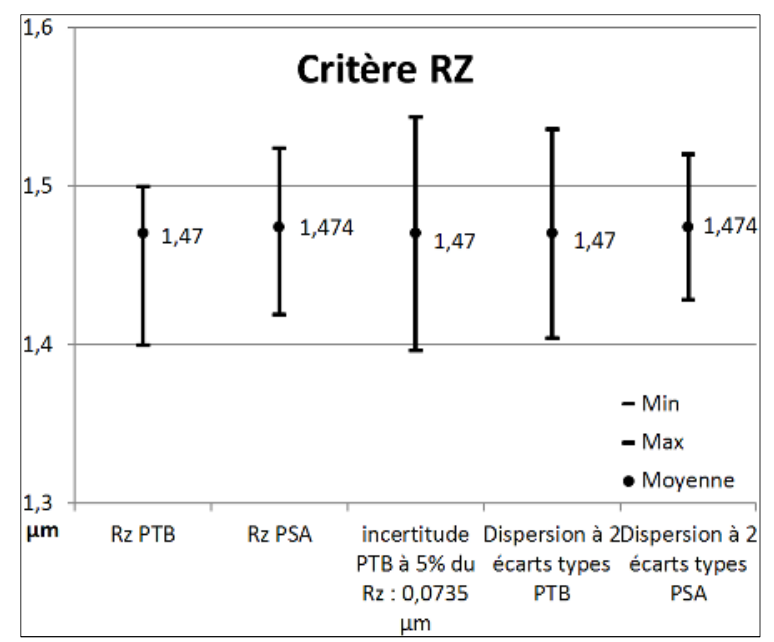

$\underline{\text { Figure 6 : }}$ Mesure du critère Rz sur l'étalon $\mathrm{N}^{\circ} 1$

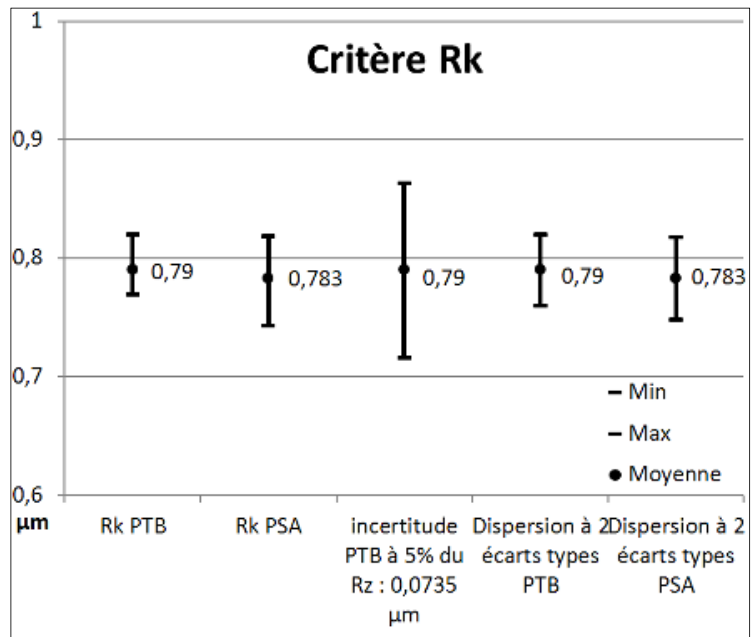

Figure 7 : Mesure du critère Rk sur l'étalon $\mathrm{N}^{\circ} 1$

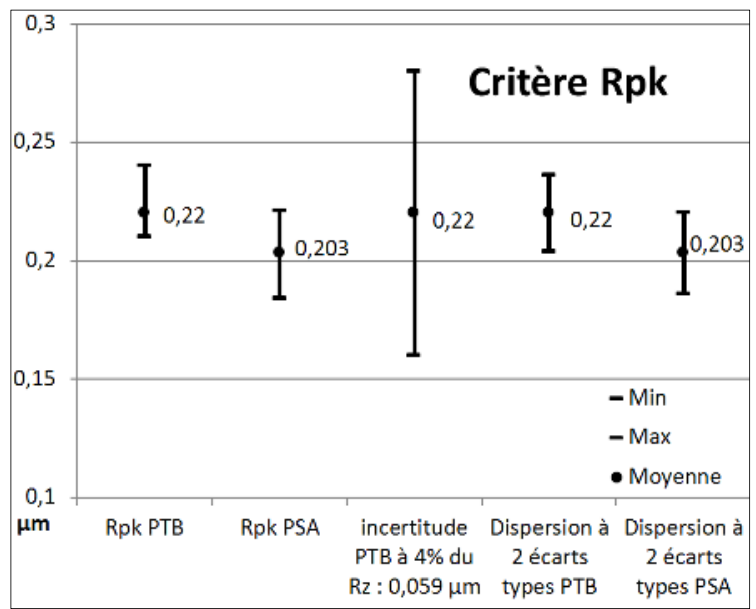

Figure 8 : Mesure du critère Rpk sur l'étalon $\mathrm{N}^{\circ} 1$

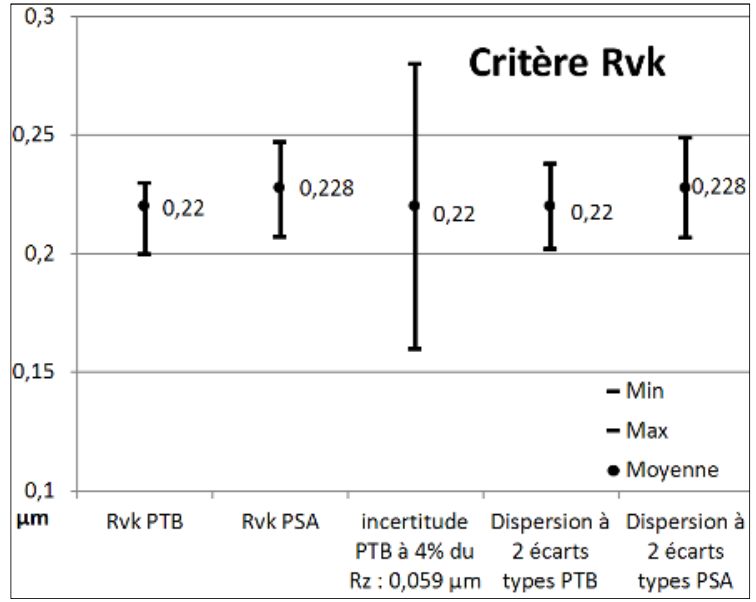

Figure 9 : Mesure du critère Rvk sur l'étalon $\mathrm{N}^{\circ} 1$

\section{Premier constat :}

Aucune de nos valeurs ne sort de l'incertitude annoncée par le certificat du PTB. Notre dispersion à deux écarts types est elle aussi toujours dans l'incertitude donnée par le certificat. 


\section{Deuxième constat :}

Les dispersions à deux écarts types de notre métrologie sont supérieures à celles du certificat de l'ordre de $15 \%$ sauf pour le $\mathrm{Rz}$ où nous sommes équivalents.

\section{Troisième constat :}

Les moyennes que nous avons calculées sont équivalentes à celles du certificat à l'exception du Rpk où nous sommes décalés de $10 \%$. Ce paramètre ne sera pas corrigé de cet écart systématique.

$\mathrm{Au}$ vu de ces différents éléments, nous pouvons annoncer une incertitude pour notre appareil sur cet étalon, et donc sur le vilebrequin égale aux valeurs du certificat pour chaque paramètre considéré.

1.6.4 Détermination de la valeur mesurée maximale acceptable pour garantir la conformité de la pièce.

Pour le paramètre Rk qui va nous servir d'exemple, la valeur maximale autorisée est de $0,3 \mu \mathrm{m}$.

Suite aux très nombreuses mesures effectuées sur notre vilebrequin de référence et sur d'autres vilebrequins, nous avons déduit que sur chaque cylindre prit individuellement la répartition des valeurs de Rk suit une loi qui est proche d'une loi uniforme.

Dans notre observation des mesures et du process de fabrication, nous avons déterminé que celui-ci ne permettait pas d'avoir, pour le paramètre Rk, des valeurs inférieures à $0,15 \mu \mathrm{m}$. Cela est dû par exemple à la taille du grain de toilage.

A partir de ces deux postulats, nous avons cherché à déterminer à travers un test statistique la valeur maximale admissible en production pour garantir la conformité de la pièce. Nous sommes en protection client.

\section{Calcul :}

Incertitude et conformité pour un caractère spécifié par un maximum.

\section{Soit :}

$\theta^{\min }$ est la valeur minimale pouvant être produite $\theta^{o b j}$ est la valeur maximale admise (tolérance) $\alpha$ est le risque décisionnel de première espèce $n$ est le nombre de mesures
On suppose que la caractéristique d'intérêt $Y$ suit une loi Uniforme $\left(\theta^{\min }, \theta\right)$ où $\theta>\theta^{\min }$ et $\theta^{\text {min }}$ représente une valeur minimale fixée. Alors :

$$
Y-\theta^{\text {min }} \sim \text { Uniforme }\left(0, \theta-\theta^{\min }\right) \text {. }
$$

Le paramètre d'intérêt est $\theta$ et c'est sur lui que porte l'expression de la conformité de la caractéristique.

Pour $\boldsymbol{Y}=\left(Y_{i}\right)_{i=1 \ldots n}$ un échantillon d'effectif $n$ indépendant identiquement distribuée de loi Uniforme $\left(\theta^{\min }, \theta\right)$,

$$
\begin{gathered}
\operatorname{Max}\left(\boldsymbol{Y}-\theta^{\text {min }}\right)=\operatorname{Max}(\boldsymbol{Y})-\theta^{\text {min }} \\
\text { et : } \\
\forall y \in\left[\theta^{\text {min }}, \theta\right] P(\operatorname{Max}(\boldsymbol{Y}) \leq y) \\
=P\left(\operatorname{Max}(\boldsymbol{X}) \leq y-\theta^{\text {min }}\right) \\
=\left(\frac{y-\theta^{\text {min }}}{\theta-\theta^{\min }}\right)^{n} \\
E(\operatorname{Max}(\boldsymbol{Y}))=\theta^{\text {min }}+\frac{n\left(\theta-\theta^{\text {min }}\right)}{n+1} \\
=\frac{1}{n+1} \theta^{\text {min }}+\left(1-\frac{1}{n+1}\right) \theta \\
V(\operatorname{Max}(\boldsymbol{Y}))=\frac{n}{(n+2)(n+1)^{2}}\left(\theta-\theta^{\text {min }}\right)^{2}
\end{gathered}
$$

Estimation de $\theta$ :

$$
\frac{n+1}{n}\left(\operatorname{Max}(\boldsymbol{Y})-\frac{1}{n+1} \theta^{\min }\right)
$$

est une estimation sans biais de $\theta$ et un intervalle de confiance de ce paramètre au niveau $1-\alpha$ est donné par :

$$
\left[\theta^{\min }+\frac{\operatorname{Max}(\boldsymbol{Y})-\theta^{\min }}{\left(1-\frac{\alpha}{2}\right)^{\frac{1}{n}}} ; \theta^{\min }+\frac{\operatorname{Max}(Y)-\theta^{\min }}{\left(\frac{\alpha}{2}\right)^{\frac{1}{n}}}\right] .
$$

Test de conformité : $H_{0}: \theta>\theta^{o b j}$ (en protection client) où $\theta^{o b j}$ est une limite fixée (tolérance).

La caractéristique est dite conforme si l'on refuse l'hypothèse nulle $H_{0}$.

Equation décisionnelle :

$$
\alpha=P\left(\operatorname{Max}(\boldsymbol{Y}) \leq k / \theta=\theta^{o b j}\right)=\left(\frac{k^{k-\theta^{\min }}}{\theta^{o b j}-\theta^{\min }}\right)^{n} .
$$

Par conséquent :

$$
\begin{aligned}
k= & \theta^{m i n}+\alpha^{\frac{1}{n}}\left(\theta^{o b j}-\theta^{m i n}\right)= \\
& \left(1-\alpha^{\frac{1}{n}}\right) \theta^{m i n}+\alpha^{\frac{1}{n}} \theta^{o b j} .
\end{aligned}
$$


La valeur maximale mesurée doit être inférieure à $\mathrm{k}$ pour que la pièce soit déclarée conforme.

\section{Application numérique :}

Sur chaque palier à mesurer, nous effectuons trois mesures, nous nous accordons un risque de $10 \%$. La limite est fixée à $0,3 \mu \mathrm{m}$.

Nous trouvons $0,220 \mu \mathrm{m}$. Ainsi, si une seule des trois valeurs mesurées dépasse ce seuil, la pièce est jugée non conforme et est rejetée.

Le seuil avec neuf mesures dans la méthode de mesure précédente était de $0,266 \mu \mathrm{m}$.

Même si le seuil baisse fortement entre les deux méthodes, les résultats de production dont nous disposons nous amènent à être confiants sur notre méthode de mesure.

\section{Mesure de volume de trous dans une chemise de carter cylindres obtenue par projection de métal}

En général dans un moteur, la chemise dans laquelle coulisse le piston est une pièce rapportée pendant ou après la coulée de la pièce. Dans cette étude, celle-ci est obtenue par projection de métal en fusion directement sur le carter cylindres. Cette technologie crée des porosités qui servent de réserve d'huile et qui sont donc fonctionnelles pour le moteur.

\subsection{Problématique :}

Déterminer la méthode de mesure et le type de moyen de mesure pour obtenir les informations suivantes :

Le volume des trous, leurs tailles et leur répartition.

\subsection{Contraintes :}

Evaluer la possibilité de suivre ces critères avec les moyens existants présents directement sur le poste de contrôle attenant à la machine de rodage, poste qui n'est pas en cabine.

Les moyens existant sont des moyens spécifiques pour la mesure des futs, à contact, le moyen reposant directement sur le haut du fût.

Pour évaluer la possibilité de mesurer avec un moyen 2D à contact, nous avons mesuré une portion de la surface avec un appareil optique de type confocal.
Puis nous avons ensuite cherché à évaluer les différences avec le moyen à contact.

\section{Premier constat :}

L'appareil optique que nous avons utilisé n'avait pas une résolution en $\mathrm{X}$ et $\mathrm{Y}$ équivalente à ce qui est demandé dans la norme $2 \mathrm{D}(0,5 \mu \mathrm{m})$. Celle-ci était de $1,56 \mu \mathrm{m}$ alors que celle de notre moyen à contact est de $0,25 \mu \mathrm{m}$. Pour les caractéristiques des trous, nous avons décidé que cette différence n'avait pas d'impact sur le résultat.

\section{Deuxième constat :}

L'appareil optique mesure jusqu'à une pente du profil maximum de $85^{\circ}$ alors que notre moyen à contact est limité à $45^{\circ}$ (pointe du stylet à $90^{\circ}$ ) nous avons donc une distorsion importante du résultat. Une évaluation mathématique théorique de cette différence n'a pas été possible car les trous n'ont pas une forme prédéterminée. Nous savons de plus que certains appareils à contact lorsqu'ils rencontrent une pente latérale importante ont tendance à dévier de leur trajectoire et donc à modifier légèrement la valeur mesurée.

\section{Troisième constat :}

Les appareils dont nous disposons en usine ne font pas de mesure 3D. Les trous n'ayant pas une orientation ou une forme particulière, il est possible théoriquement de faire un rapport entre volume des trous par unité de surface et la surface des trous par unité de longueur. Cependant la forte non homogénéité de la surface rend cette tâche difficile.

\subsection{Influence de l'application de la norme dans le résultat de mesure 2D à contact}

Nous avons rencontré un problème inattendu lors de la mesure des pièces avec les moyens à contact classique de notre métrologie. Pour le calcul des paramètres Rk Rpk et Rvk nous suivons les prescriptions de la norme ISO 13565 qui nous oblige à utiliser un filtre gaussien normalisé (ISO 16610-21 anciennement ISO 11562) et nous conseille d'utiliser un $\lambda \mathrm{c}$ de $0,8 \mathrm{~mm}$ voire de $2,5 \mathrm{~mm}$ dans certains cas.

Nous avons constaté lors de nos premières mesures que ce filtre, qui n'est pas très performant, crée des distorsions dans la mesure lors de la présence de trous importants malgré le double filtrage de cette norme. L'augmentation du $\lambda \mathrm{c}$ n'a résolu que partiellement le problème.

Nous avons décidé de déroger à la norme en utilisant un filtre gaussien robuste qui nous a permis de 
résoudre le problème, avec cependant plusieurs difficultés : ce filtre n'est pas présent sur de nombreux appareils et de plus, ces appareils ne peuvent contourner la norme. Pour les logiciels vendus hors matériels et contrairement au filtre gaussien de l'ISO 16610-21 qui est parfaitement connu, les filtres gaussien robuste de l'ISO 16610-31 ne sont pas complètements définis et donc entrainent une incertitude logicielle.

Nos moyens d'usines ne disposant pas, à ce jour, de capacité pour traiter les résultats avec le filtre gaussien robuste, nous avons traité les mesures dans notre métrologie centrale.

\subsection{Etude comparative entre mesure surfacique et mesure linéaire, à contact et sans contact.}

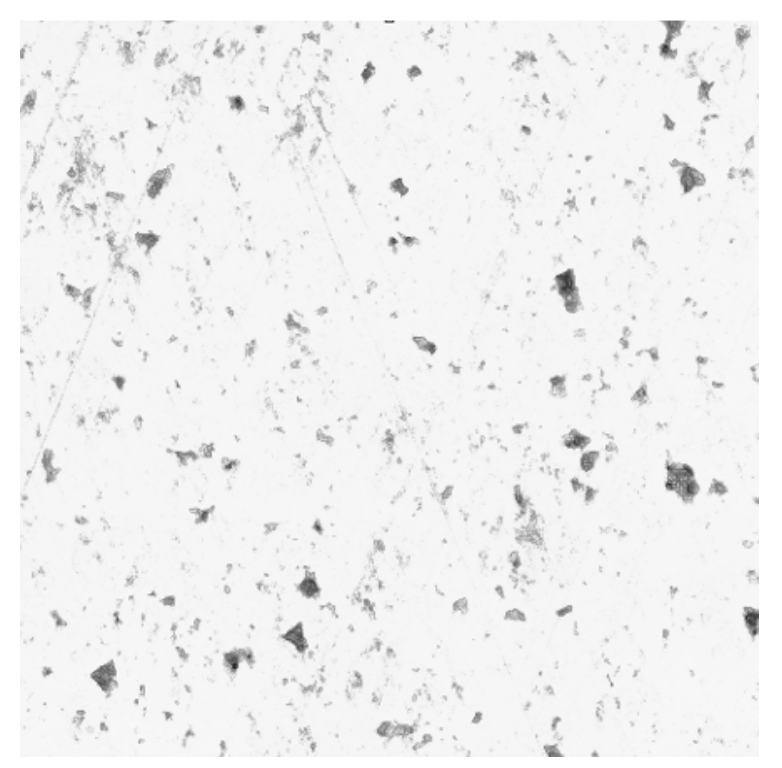

\section{Premier constat :}

Il y a $15 \%$ d'écart sur le volume des trous entre la mesure 3D optique et la mesure 3D à contact (cette mesure ne peut être effectue en usine mais est réalisable dans notre métrologie) l'appareil optique trouvant un volume de trous plus important.

\section{Deuxième constat :}

Si l'on cherche à faire une corrélation entre le volume de trous obtenus en 3D et la surface de trous obtenus sur une ligne, on constate que $95 \%$ des valeurs de trous pour une ligne sont comprises entre une valeur proche de zéro et le double de la valeur $\mathrm{du}$ volume que l'on trouve en $3 \mathrm{D}$ et ce de façon relativement uniforme.

\section{Troisième constat :}

Si l'on tente de faire une comparaison ligne à ligne entre la mesure optique et la mesure à contact, on constate que la différence de valeur de $15 \%$ entre contact et optique n'est pas fixe et varie même fortement en fonction de la typologie des trous rencontrés.

\section{Quatrième constat :}

La variation entre deux zones mesurées en $3 \mathrm{D}$ de même taille est de $+/-15 \%$.

\section{Cinquième constat :}

Pour calculer le volume de trous sur la mesure 3D nous utilisons le critère $\mathrm{Vvv}$ qui est le volume de vide des creux de la surface

Pour calculer la surface des creux d'un profil nous utilisons le critère Rpk.

Ces deux critères, même s'ils sont les plus approchants entre $2 \mathrm{D}$ et $3 \mathrm{D}$, entrainent une erreur de calcul que nous n'avons pas réussi à évaluer.

\subsection{Conclusions :}

Nous avons montré que le respect de la norme ISO13565 peut entrainer une erreur sur le résultat des critères Rk, Rpk et Rvk.

Nous avons montré qu'il est possible de contourner ce problème en dérogeant à la norme.

Nous avons estimé l'écart entre une mesure 3D à contact et $3 \mathrm{D}$ sans contact. Compte tenu du faible échantillon de pièces dont nous disposons nous ignorons la fiabilité de ce résultat.

Nous avons montré que la surface était fortement non homogène vis-à-vis de la répartition, de la taille et de la forme des trous. Ainsi si nous savons déterminer le volume de trous de façon fiable avec un nombre raisonnable de mesures $2 \mathrm{D}$, il n'est pas possible d'en faire autant pour la répartition, la taille et la forme des trous. Ce constat nous amène donc à rejeter nos moyens actuels pour le suivi de ces critères. L'incertitude de méthode serait trop importante.

Pour ce qui est de la mesure de la rugosité hors trous, nos matériels actuels donnent satisfaction moyennant certaines adaptations. Ces moyens pourront continuer d'assurer le suivi des moyens de fabrication pour la partie rodage.

Ces nouveaux moyens optiques en $3 \mathrm{D}$ ne pourront être localisés directement dans la ligne de fabrication à cause des conditions environnementales de celle-ci. 